\title{
Acute coronary syndromes: consensus recommendations for translating knowledge into action
}

David Brieger, Anne-Maree Kelly, Constantine Aroney, Philip Tideman, Saul B Freedman, Derek Chew, Marcus Ilton, Gerard Carroll, Ian Jacobs and Nancy P Huang on behalf of the National Heart Foundation ACS Implementation and Advocacy Working Group

T he Australian Institute of Health and Welfare estimates that 48700 coronary heart disease (CHD) events (deaths or hospitalisations) occurred among Australians aged 40-90 years in 2001-2002. ${ }^{1}$ In 2007, acute myocardial infarction was the underlying cause of 11341 deaths. ${ }^{2}$ It is estimated that in 2009, over 50000 Australians will have an acute myocardial infarction, at a cost of $\$ 281000$ to the community for each event. ${ }^{3}$

National guidelines for the management of acute coronary syndromes (ACS), ${ }^{4}$ published in 2006 by the National Heart Foundation of Australia and the Cardiac Society of Australia and New Zealand, provided recommendations based on practices that have been shown to improve outcomes for patients with ACS. 5,6 The ACS Implementation and Advocacy Working Group was established by the Heart Foundation to identify ways to enhance uptake of the guidelines. Here, we describe the development of consensus recommendations to improve the delivery of key aspects of clinical care set out in the guidelines.

\section{Process}

In 2007, the Heart Foundation, in collaboration with the Cardiac Society of Australia and New Zealand and the Australasian College for Emergency Medicine, held a national forum ${ }^{7}$ to promote implementation of the 2006 national ACS management guidelines. ${ }^{4}$ The forum brought together representatives of key stakeholder organisations and key opinion leaders from the areas of clinical cardiology, nursing, cardiac rehabilitation, emergency medicine, government, general practice and rural health. Its aims were to identify key gaps in the current management of ACS and develop a set of priority actions that would achieve the greatest improvement in outcomes among ACS patients. ${ }^{7}$

Forum workshops used the nominal group technique, a moderated group process that allows each participant to voice and then vote on identified priorities, ${ }^{8}$ to reach consensus on priority intervention points in four areas of ACS management:

- early reperfusion for patients with ST-segment-elevation myocardial infarction (STEMI);

- risk stratification for patients with non-ST-segment-elevation ACS (NSTEACS);

- access to invasive procedures for patients with high- and intermediate-risk NSTEACS; and

- postacute care, including discharge therapies, access to rehabilitation services, and the interface between acute and primary care services.

Members of the ACS Implementation and Advocacy Working Group undertook a literature review to identify gaps between guidelines and current practice, and evidence for the most effective systems of ACS management. After the forum, the Working Group developed a set of recommendations for implementation, based on the priority interventions identified at the forum. ${ }^{7}$

\section{ABSTRACT}

- A systematic, integrated national approach is needed to implement 2006 Australian guidelines for management of acute coronary syndromes (ACS). Clinical outcomes can be improved by closing the current gaps between evidence and practice.

- In 2007, the National Heart Foundation of Australia, the Cardiac Society of Australia and New Zealand, and the Australasian College for Emergency Medicine held a national forum to identify current gaps in ACS management and priority strategies to improve outcomes. Consensus recommendations were based on evidence and expert opinion.

- Prompt reperfusion for patients with ST-segment-elevation myocardial infarction should be ensured by establishing protocols for single-call activation of primary percutaneous coronary intervention, or, where unavailable, enabling health care workers to initiate thrombolysis.

- Accuracy of risk stratification of non-ST-segment-elevation ACS (NSTEACS) should be improved using clinical pathways that integrate ambulance, medical and nursing care.

- Rates of early invasive management for patients with high-risk NSTEACS should be increased using efficient systems for transfer to revascularisation facilities.

- All patients with an ACS should be referred to rehabilitation and secondary prevention programs, including alternative models of care where appropriate.

- Equal access to recommended care for all Australians with an ACS — including those in rural, remote and Aboriginal and Torres Strait Islander communities — should be achieved by improving workforce capacity in under-resourced regions and ensuring access to third-generation fibrinolytic agents, defibrillation, timely essential pathology tests and invasive revascularisation facilities.

- National standards for data collection and clinical outcomes should be established, and performance should be monitored.

MJA 2009; 191: 334-338

\section{Outcomes}

The ACS Implementation and Advocacy Working Group proposed recommendations in six key priority areas, each of which will be promoted through implementation activities planned for 20092010 (Box)

\section{Early reperfusion for STEMI}

Current guidelines recommend early reperfusion for patients with STEMI. ${ }^{4}$ The earlier the delivery of reperfusion, the better the outcomes. ${ }^{9}$ If delivered promptly (within 90 minutes of presentation in most settings), percutaneous coronary intervention (PCI) 


\section{Summary of priority areas and intended actions of the National Heart Foundation of Australia ACS Implementation and Advocacy Working Group for 2009-2010}

\section{Recommendations}

\section{Early reperfusion for STEMI}

Empower and support appropriately trained health care workers to initiate thrombolysis in regional and remote settings. Set up protocols for single-call activation of $\mathrm{PCl}$ systems that operate 24 hours a day, 7 days a week. Optimise the use of existing infrastructure for reperfusion through regional collaborations and protocols.
Actions

Develop a national web-based portal to enable health services and clinical networks to access and share locally developed evidence-based clinical pathways and educational tools.

\section{Accurate risk stratification for NSTEACS}

Implement clinical pathways that incorporate risk stratification, treatment and referral, and are adapted to local conditions and sponsored by executive management and clinicians. Include risk stratification measurement and feedback in KPIs (see priority area 6). Provide health care workers with appropriate training in risk assessment patient monitoring and interpretation of electrocardiograms and troponin tests.

\section{Early invasive management for high-risk NSTEACS}

Ensure that all Australians have timely access to appropriate invasive revascularisation facilities by establishing sufficient catheterisation laboratories, based on a hub-and-spoke network model with clear referral pathways.

\section{Rehabilitation and secondary prevention}

Set up systems of prompts and recalls to ensure that all patients are offered referrals to cardiac rehabilitation or alternative programs.

Deliver programs outside business hours, or through health professional home visits. Expand alternative secondary prevention programs in addition to traditional cardiac rehabilitation programs.
Develop an electronic decision support tool to support evidence-based care and workflow integration throughout the acute health care setting. Facilitate exchange of locally developed tools and clinical pathways for risk stratification (see priority area 1).

\section{Equality of access and care for geographically isolated and vulnerable communities}

Ensure all centres treating patients with ACS have access to defibrillation, timely on-site pathology, and expertise to deliver thrombolysis, including third-generation fibrinolytic agents (see priority area 1). Address workforce capacity issues in under-resourced regions.

\section{Data collection and programs for improving clinical effectiveness}

Establish a minimum dataset for ACS management. Develop tools and resources that will support the local collection of these data. Develop KPIs and set up systems for reporting and feedback through standard health department processes.
Advocate adequate planning and resource allocation at national and state levels to ensure sufficient density of catheterisation laboratories. Facilitate exchange of locally developed tools and clinical pathways for triage and interhospital transfer (see priority area 1).

Advocate dedicated national and state-based funding to support the expansion of cardiac rehabilitation and secondary prevention services for geographically isolated and vulnerable communities (see priority area 5). drive systemic change, reduce hospital disparities and improve ACS outcomes for all, including Aboriginal and Torres Strait Islander peoples and people living in rural and remote regions. Support studies to explore barriers to guideline adoption in regional and remote communities.

Specifically advocate, to government and policymakers, a range of outcome measures and data initiatives to drive quality improvement systems for people with ACS. Develop a position statement for publication on data requirements for improved ACS management in Australia.

$\mathrm{ACS}=$ acute coronary syndromes. $\mathrm{KPI}=$ key performance indicator. NSTEACS $=$ non-ST-segment-elevation acute coronary syndromes. PCI = percutaneous coronary intervention. STEMI = ST-segment-elevation myocardial infarction.

achieves superior outcomes to those achieved with fibrinolysis. ${ }^{10}$ Where PCI is not available, fibrinolysis is the best option, and should be delivered within 30 minutes of presentation to hospital, or in the prehospital setting if feasible. ${ }^{4,11}$

Current management gaps. Recent Australian registry data ${ }^{12}$ show that:

- median presentation-to-PCI interval is 102 minutes, with the benchmark of 90 minutes exceeded in $65.7 \%$ of patients;

- among patients treated with a fibrinolytic agent, the median presentation-to-needle time is 45-68 minutes, with the benchmark of 30 minutes exceeded in most patients; and

- up to $30 \%$ of patients with STEMI do not receive reperfusion therapy; this is comparable with observations in other developed countries. $^{13}$
Strategies for closing these gaps. Effective strategies to reduce delay between presentation and reperfusion include systems to:

- enable the catheterisation laboratory to be activated by a single call by the first health care professional qualified to recognise STEMI, bypassing traditional hospital hierarchical decision-making systems; ${ }^{14,15}$

- activate the catheterisation laboratory before the patient arrives at the hospital, ${ }^{15}$ either through an ambulance-based 12-lead electrocardiogram (ECG) or a voice call; ${ }^{16}$

- transfer patients directly to a reperfusion-capable facility in preference to a closer non-reperfusion centre ${ }^{16}$ and

- enable initiation of fibrinolytic therapy by health care workers other than doctors, including in a prehospital setting in some circumstances. ${ }^{11}$ 


\section{POSITION STATEMENT}

No single system will improve reperfusion rates and times in all Australian regions. Modelling studies are needed to ensure patients in all geographical locations have timely access to reperfusioncapable facilities, either through more efficient use of existing resources or by setting up additional centres. Collaboration between local agencies is recommended so that the optimal system for each region can be developed based on case loads, resources, workforce, geographical considerations and referral patterns.

\section{Accurate risk stratification for NSTEACS}

Once ST-elevation has been excluded, guidelines recommend formal risk stratification of patients with suspected NSTEACS to identify those at high risk of an adverse cardiac outcome. This process involves a detailed history, physical examination, and interpretation of electrocardiograms and blood tests, including troponin assays. ${ }^{4}$ Decision-making tools, such as clinical pathways and ACS protocols, have been shown to improve the accuracy of risk stratification and referral to appropriate evidence-based therapies. ${ }^{17,18}$ Clinical pathways are agreed procedures for applying protocols in the local setting, including prehospital, intra- and interhospital transfer of patients. Components may include risk stratification tools for identifying patients who belong to low-, moderate- or high-risk categories to guide clinical management. ACS protocols are standardised procedures for applying evidencebased guidelines at the state, network or institution levels, to guide the "rules of business" for ACS management.

Current management gaps. Evidence of discordance between assessed risk and intensity of management ${ }^{19}$ suggests that risk stratification for patients with ACS is often not performed appropriately. Furthermore, resistance to the use of clinical algorithms has been observed in some Australian hospitals. ${ }^{20}$

Strategies for closing these gaps. The process of risk stratification can be improved by:

- implementing clinical pathways that incorporate triage, risk assessment and referral, taking into account local context. This would require clinical leadership, education, and acceptance of the agreed clinical pathway by medical staff, on the understanding that it is not intended to replace clinical judgement;

- a system-wide approach to staff training to overcome barriers encountered due to mobility of the medical workforce; and

- including risk stratification measurement and feedback in key performance indicators (KPIs) (see priority area 6).

\section{Early invasive management for high-risk NSTEACS}

Guidelines recommend access to coronary angiography and $\mathrm{PCI}$ for patients with high-risk NSTEACS, ${ }^{4}$ based on evidence that the use of these interventions improves outcomes. ${ }^{21-24}$

Current management gaps. Available Australian data indicate that the probability of patients with high-risk NSTEACS receiving angiography and PCI or coronary artery bypass graft (CABG) surgery is significantly reduced when they present to a hospital without angiographic facilities, and that the incidence of this situation has increased since the early 1990s. ${ }^{23,25,26}$ Although some regions face a significant lack of resources, existing resources and facilities are not being fully utilised in others. For instance, recent New South Wales health data indicate that revascularisation rates can vary by up to $30 \%$ between area health care services with comparable numbers of catheterisation facilities. ${ }^{27}$
Strategies for closing these gaps. Increased access to invasive management for patients with high-risk NSTEACS will require a combination of decentralisation of invasive facilities ${ }^{3}$ and systembased approaches to overcome other barriers to access. Further feasibility modelling will be required to determine the population needed to support a catheterisation laboratory in each region. A hub-and-spoke model of hospital networks is an effective mechanism for improving access. This requires attention to:

- triage systems (see priority area 2);

- systems of communication between clinical staff, departments and institutions; and

- patient transport between facilities.

These systems must be tailored for local conditions, particularly in rural and remote settings (see priority area 5).

\section{Rehabilitation and secondary prevention}

Current guidelines recommend that all patients with ACS be referred to a cardiac rehabilitation program following an acute event. ${ }^{4}$ Recent data from the United States and the United Kingdom suggest that secondary prevention therapies have been as significant as acute therapies in achieving the reduction in ageadjusted CHD mortality seen over the past two decades. ${ }^{28,29}$

Current management gaps. Only about 30\% of eligible Australian patients access cardiac rehabilitation programs, comparable with findings overseas, ${ }^{30,31}$ and this has not improved significantly over the past 10 years. ${ }^{26}$ Failure to educate patients about the importance of secondary prevention strategies probably contributes to the 10\%-15\% medication drop-off rates observed in the 12 months following hospital discharge. ${ }^{23,26}$

Strategies for closing these gaps. Participation in cardiac rehabilitation or secondary prevention programs after ACS can be improved by:

- implementing protocols for referral to cardiac rehabilitation programs for all ACS patients, including those who had NSTEACS or have undergone CABG surgery;

- offering cardiac rehabilitation program sessions on evenings and weekends or through health professional home visits; and

- expanding effective alternative secondary prevention programs such as Choice of Health Options In prevention of Cardiovascular Events (CHOICE) ${ }^{32,33}$ and Coaching patients On Achieving Cardiovascular Health $(\mathrm{COACH})$, which have been shown to be effective in reducing risk in the Australian setting. ${ }^{34,35}$

\section{Equality of access and care for geographically isolated and vulnerable communities}

Although most Australians live in urban centres, 10\%-30\% (depending on definition) live in rural and remote areas. Where the nearest PCI centre is over 90 minutes away, thrombolysis becomes the choice for reperfusion therapy. All centres currently treating ACS need access to third-generation single-dose fibrinspecific fibrinolytic agents (tenecteplase or reteplase).

Current management gaps. The yearly CHD-related death rate increases with remoteness, from 71.1 per 100000 population in metropolitan areas to 85.5 in remote Australia. ${ }^{36}$ Aboriginal and Torres Strait Islander peoples experience higher mortality rates from CHD than other Australians. ${ }^{1}$ Health system issues differ between urban, rural and remote Aboriginal and Torres Strait Islander communities. 


\section{POSITION STATEMENT}

Despite guideline recommendations against the use of streptokinase, it is still used in some centres, particularly regions with Indigenous populations. The greatest efficiencies in remote settings are achieved where local health care workers are able to deliver fibrinolysis. However, staff in remote areas may lack appropriate training and access to expert support for decision making. There is some evidence that Aboriginal and Torres Strait Islander peoples may not receive ideal care once hospitalised. ${ }^{37} \mathrm{~A}$ shortage of culturally appropriate health care workers in these facilities probably contributes to this problem. Failure to provide secondary prevention for patients presenting to rural and remote locations has also been reported. ${ }^{38}$

Strategies for closing these gaps. ACS care for geographically isolated or vulnerable communities can be improved by:

- ensuring that hospitals, community health centres and medical centres have access to point-of-care pathology and electrocardiography, and technology for electronic transfer of ECG images;

- developing transparent and culturally appropriate regional systems for prompt and appropriate patient transfer of high-risk patients; ${ }^{23,26}$ and

- developing local networks that are identifiable to local communities

\section{Data collection and programs for improving clinical effectiveness}

A nationally standardised collection of clinical data, with feedback on patient characteristics, processes of care and clinical outcomes, would enable comparisons within and between health care facilities, and inform improvements in clinical effectiveness. ${ }^{39}$

Current management gaps. There is a lack of standardised information on the care of patients with ACS across the continuum of ACS management in Australia. Available evidence indicates that ACS management in Australia is suboptimal, consistent with findings from other developed nations. ${ }^{40}$

Strategies for closing these gaps. Systems for optimal data collection and governance should be established, with:

- standards for a minimum dataset including patient characteristics, investigations, management and clinical outcomes;

- development of KPIs;

- mandated audit of data veracity for 10\%-15\% of clinical presentations;

- reporting of KPIs through standard health department processes, and aggregation of data at state and national levels; and

- communication of performance outcomes to hospitals and clinical networks, with appropriate peer group comparisons.

Local health facilities should be responsible for collection of data, and retain ownership. Incentives to participate in this process may be facilitated by the development of multisite quality improvement collaboratives. State and federal governments should be responsible for aggregating data at state and national levels, respectively.

\section{Implementation}

If the quality of care for patients with ACS is to be improved, current inequities addressed and the likely future increase in demand met, Australia must adopt a systemic approach. This will involve implementation of evidence-based protocols for risk assessment, timely reperfusion and ongoing care, all of which must be adapted to local conditions. These efforts must be underpinned by national outcome standards, with scope for regional flexibility in effective service delivery, and agreed processes for data collection and monitoring. There should be a focus on providing services to regional, rural and remote environments, and on addressing the specific requirements of Aboriginal and Torres Strait Islander communities.

Responsibility for planning, implementing and funding such an approach rests with governments, but professional associations, such as those involved in the recent ACS implementation forum, bear responsibility for clinical leadership and training. By working together and engaging key stakeholders, these professional bodies are committed to a course of improving ACS care around Australia.

\section{Competing interests}

Constantine Aroney has received consultancy fees, speaker fees or travel assistance from The Medicines Company, Sanofi-Aventis and Pfizer. Gerard Carroll has received speaker fees for educational lectures to doctors, and has received travel assistance to several international cardiovascular meetings from Pfizer, Astra Zeneca, MSD and Sanofi-Aventis. Derek Chew is employed by Flinders University through a position that is supported by a Heart Foundation Grant.

\section{Author details}

David Brieger, PhD, FRACP, Cardiologist ${ }^{1}$ Anne-Maree Kelly, MD BS, MClinED, FACEM, Director $^{2}$

Constantine Aroney, MD, FRACP, Director, Cardiac Services ${ }^{3}$ Philip Tideman, FRACP, Cardiologist ${ }^{4}$ and Clinical Director ${ }^{5}$

Saul B Freedman, PhD, FRACP, FCSANZ, Professor of Cardiology, ANZAC Research Institute, ${ }^{1}$ and Deputy Dean ${ }^{6}$

Derek Chew, MB BS, MPH, FRACP, Acting Director, Cardiovascular Services, ${ }^{4}$ and Leader, Clinical Change Area of Strategic Research Investment ${ }^{7}$

Marcus Ilton, MB BS, FRACP, Cardiologist ${ }^{8}$

Gerard Carroll, AM, PhD, FACAP, Cardiologist ${ }^{9}$

Ian Jacobs, PhD, FACAP, RN, Head, ${ }^{10}$ Chairman, ${ }^{11}$ and Director ${ }^{12}$

Nancy P Huang, MB BS, DipRACOG, MPH, National Manager Clinical Programs ${ }^{13}$

1 Concord Repatriation General Hospital, Sydney, NSW.

2 Joseph Epstein Centre for Emergency Medicine Research,

Western Health, Melbourne, VIC.

3 Holy Spirit Northside Private Hospital, Brisbane, QLD.

4 Flinders Medical Centre, Adelaide, SA.

5 Integrated Cardiovascular Clinical Network SA, Adelaide, SA.

6 Faculty of Medicine, University of Sydney, Sydney, NSW.

7 Flinders Centre for Clinical Change and Health Care Research,

Flinders University, Adelaide, SA.

8 Royal Darwin Hospital, Darwin, NT.

9 Wagga Wagga Base Hospital, Wagga Wagga, NSW.

10 Department of Emergency Medicine, University of Western Australia, Perth, WA.

11 Western Australian Branch, Australian Resuscitation Council, Perth, WA.

12 Western Australian Prehospital Care Research Unit, Perth, WA.

13 National Heart Foundation of Australia, Melbourne, VIC.

Correspondence: davidb@email.cs.nsw.gov.au

\section{References}

1 Australian Institute of Health and Welfare. Heart, stroke and vascular diseases, Australian facts 2004. Canberra: AlHW and National Heart Foundation of Australia, 2004. (AlHW Cat. No. CVD 27. Cardiovascular Disease Series No. 22.)

2 Australian Bureau of Statistics. Causes of death 2007. Canberra: ABS, 2009. (ABS Cat. No. 3303.0.) 


\section{POSITION STATEMENT}

3 Access Economics. The economic cost of heart attack and chest pain (acute coronary syndrome). Canberra: Eli Lilly, 2009. http://accesseconomics.com.au/publicationsreports/showreport.php?id=204\&searchfor= 2009\&searchby=year (accessed Jul 2009).

4 Acute Coronary Syndrome Guidelines Working Group. Guidelines for the management of acute coronary syndromes 2006. Med J Aust 2006; 184 (8 Suppl): S1-S32.

5 Scott IA, Harper CM. Guideline-discordant care in acute myocardial infarction: predictors and outcomes. Med J Aust 2002; 177: 26-31.

6 Mäkikallio TH, Barthel P, Schneider R, et al. Frequency of sudden cardiac death among acute myocardial infarction survivors with optimized medical and revascularization therapy. Am J Cardiol 2006; 97: 480-484.

7 National Heart Foundation of Australia. Summary report from the National Acute Coronary Syndromes Implementation Forum; 2007 Oct 12; Melbourne. Melbourne: Heart Foundation, 2007.

8 Evaluation Research Team, Centers for Disease Control and Prevention. Gaining consensus among stakeholders through the nominal group technique. Evaluation Briefs 2006; Nov (7). http://www.cdc.gov/Healthy Youth/evaluation/pdf/brief7.pdf (accessed Aug 2009).

9 White HD, Chew DP. Acute myocardial infarction. Lancet 2008; 372: 570584.

10 Keeley EC, Boura JA, Grines CL. Primary angioplasty versus intravenous thrombolytic therapy for acute myocardial infarction: a quantitative review of 23 randomised trials. Lancet 2003; 361: 13-20.

11 Steg PG, Bonnefoy E, Chabaud S, et al. Impact of time to treatment on mortality after prehospital fibrinolysis or primary angioplasty: data from the CAPTIM randomized clinical trial. Circulation 2003; 108: 2851-2856.

12 Erickson M, Rankin J, Amerena J, et al. Reperfusion therapy in STEMI: ACACIA registry contemporary Australian data [abstract]. Heart Lung Circ 2007; 16 Suppl 2: S124

13 Eagle KA, Goodman SG, Avezum A, et al. Practice variation and missed opportunities for reperfusion in ST-segment-elevation myocardial infarction: findings from the Global Registry of Acute Coronary Events (GRACE). Lancet 2002; 359: 373-377.

14 Krumholz HM, Bradley EH, Nallamothu BK, et al. A campaign to improve the timeliness of primary percutaneous coronary intervention: Door-toBalloon: An Alliance for Quality. JACC Cardiovasc Interv 2008; 1: 97-104.

15 Bradley EH, Herrin J, Wang Y, et al. Strategies for reducing the door-toballoon time in acute myocardial infarction. N Engl J Med 2006; 355: 2308-2320

16 Brown JP, Mahmud E, Dunford JV, Ben-Yehuda O. Effect of prehospital 12-lead electrocardiogram on activation of the cardiac catheterization laboratory and door-to-balloon time in ST-segment elevation acute myocardial infarction. Am J Cardiol 2008; 101: 158-161.

17 Scott IA, Darwin IC, Harvey KH, et al. Multisite, quality-improvement collaboration to optimise cardiac care in Queensland public hospitals. Med J Aust 2004; 180: 392-397.

18 Huynh LT, Phillips PA, Chew DP. Improving the management of acute coronary syndromes in Australia: translating evidence to outcomes. Intern Med J 2007; 37: 412-415.

19 Scott IA, Derhy PH, O'Kane D, et al. Discordance between level of risk and intensity of evidence-based treatment in patients with acute coronary syndromes. Med J Aust 2007; 187: 153-159.

20 National Institute of Clinical Studies. Identifying barriers to evidence uptake. Melbourne, NICS: 2006.

21 Cannon CP, Weintraub WS, Demopoulos LA, et al. Comparison of early invasive and conservative strategies in patients with unstable coronary syndromes treated with the glycoprotein IIb/IIla inhibitor tirofiban. N Engl J Med 2001; 344: 1879-1887.

22 Fox KA, Poole-Wilson PA, Henderson RA, et al. Interventional versus conservative treatment for patients with unstable angina or non-STelevation myocardial infarction: the British Heart Foundation RITA 3 randomised trial. Randomized Intervention Trial of unstable Angina. Lancet 2002; 360: 743-751.

23 Chew DP, Amerena JV, Coverdale SG, et al. Invasive management and late clinical outcomes in contemporary Australian management of acute coronary syndromes: observations from the ACACIA registry. Med J Aust 2008; 188: 691-697.

24 Hoenig MR, Doust JA, Aroney CN, Scott IA. Early invasive versus conservative strategies for unstable angina \& non-ST-elevation myocardial infarction in the stent era. Cochrane Database Syst Rev 2006; (3): CD004815

25 Tideman PA, Fabri JK, Aylward PE, Simes RJ. Intervention with PTCA and CABG following thrombolysis for acute myocardial infarction. Australian data from GUSTO 1 (1991-3) and International Study Group r-TPAStreptokinase Mortality (1989) trials. Global Utilisation of Streptokinase and Tissue. Aust N Z J Med 1998; 28: 533-540.

26 Walters DL, Aroney CN, Chew DP, et al. Variations in the application of cardiac care in Australia. Med J Aust 2008; 188: 218-223.

27 Population Health Division. Health of the people of NSW: summary report. Report of the Chief Health Officer 2008. Sydney: NSW Department of Health, 2008.

28 Ford ES, Ajani UA, Croft JB, et al. Explaining the decrease in US deaths from coronary disease, 1980-2000. N Engl J Med 2007; 356: 2388-2398.

29 Unal B, Critchley JA, Capewell S. Explaining the decline in coronary heart disease mortality in England and Wales between 1981 and 2000. Circulation 2004; 109: 1101-1107.

30 Scott IA, Lindsay KA, Harden HE. Utilisation of outpatient cardiac rehabilitation in Queensland. Med J Aust 2003; 179: 341-345.

31 Wenger N. Current status of cardiac rehabilitation. J Am Coll Cardiol 2008; 51: 1619-1631.

32 Redfern J, Ellis E, Briffa T, Freedman SB. Patient-centered modular secondary prevention following acute coronary syndrome: a randomized controlled trial. J Cardiopulm Rehabil Prev 2008; 28: 107-115.

33 Redfern J, Briffa T, Ellis E, Freedman SB. Choice of secondary prevention improves risk factors after acute coronary syndrome: one year follow-up of the CHOICE (Choice of Health Options In prevention of Cardiovascular Events) randomised controlled trial. Heart 2009; 95: 468-475.

34 Vale MJ, Jelinek MV, Best JD, et al. COACH Study Group. Coaching patients On Achieving Cardiovascular Health (COACH): a multicenter randomized trial in patients with coronary heart disease. Arch Intern Med 2003; 163: 2775-2783

35 Jelinek $M$, Vale $M$, Dart $A$, et al. The $\mathrm{COACH}$ program produces sustained improvements in cardiovascular risk factors and adherence to recommended medications - 18 months follow-up [abstract]. Heart Lung Circ 2008; 17 Suppl 3: S203.

36 Mathur S, Moon L, Leigh S. Aboriginal and Torres Strait Islander people with coronary heart disease (summary booklet): further perspectives on health status and treatment. Canberra: Australian Institute of Health and Welfare, 2006. (AlHW Cat. No. CVD 34. Cardiovascular disease series no. 26.)

37 Coory MD, Walsh WF. Rates of percutaneous coronary interventions and bypass surgery after acute myocardial infarction in Indigenous patients. Med J Aust 2005; 182: 507-512.

38 Brieger D, Amerena S, Coverdale J, et al. Logistics of ACS management in rural versus metropolitan hospitals [abstract]. Heart Lung Circ 2007; 16 Suppl 2: S121.

39 Chew DP, Allan RM, Aroney CN, Sheerin NJ. Acute Coronary Syndrome Data Set Working Group. National data elements for the clinical management of acute coronary syndromes. Med J Aust 2005; 182 (9 Suppl): S1-14.

40 Fox KA. An international perspective on acute coronary syndrome care: insights from the Global Registry of Acute Coronary Events. Am Heart $J$ 2004; 148: S40-S45.

(Received 10 Mar 2009, accepted 23 Jul 2009) 